\title{
Occlusion of the vortex veins
}

\section{An experimental study}

\author{
S. S. HAYREH* AND J. A. B. BAINES $\dagger$ \\ Department of Ophthalmology, University of Edinburgh
}

The clinical picture of retinal venous occlusion is well known to ophthalmologists, but no corresponding description of vortex vein (VV) occlusion in man is so far available. After completing our present experimental study, we carried out an exhaustive search of the literature which revealed some old accounts of experimental occlusion of the different VVs. These were mostly designed to produce experimental glaucoma (Adamiuk, I867; Leber, I873; Weber, I877; Schoeler, I879; von Schultén, I884; Arlt, I884; Ulrich, I889; Müller, ı889; Koster, I895; Huggert, I957; Macri, 1960), in one instance to produce retinal detachment (Sachsenweger and Lukoff, I959), and in another to find out histopathological changes in the VV (Hirata, 1969). All the earlier studies were carried out in rabbits except for those of Sachsenweger and Lukoff (1959) in dogs, Adamiuk (1867) and Macri (1960) in cats, and Müller (1889) in the horse. Only Koster (1895) dealt in detail with most of the ocular signs of $\mathrm{VV}$ occlusion.

We have produced experimental occlusion of one, two, three, and all of the VVs in rhesus monkeys and have studied their short-term and long-term effects on the eye by a detailed clinical examination, intravenous fluorescein angiography, histopathological studies, and perfusion of the ocular vascular bed with silicone rubber. Since the uveal vasculature in man and rhesus monkeys is identical, it can be assumed that the findings in rhesus monkeys will hold good in man. A clinical picture of VV occlusion, derived from these studies, is presented in this paper.

\section{Material}

This study was carried out in forty eyes of rhesus monkeys.

\section{Methods}

After lateral orbitotomy the VVs were occluded by cauterizing them a short distance from their site of exit from the globe without any other interference with the ocular vessels. The details of the occlusions are shown in Table $\mathbf{I}$.

These eyes were followed up for variable periods (Table II).

In all these eyes the following investigations were carried out soon after the occlusion, later on during the follow-up period, and at the termination of the experiment. 
Table I Numbers and names of the vortex veins occluded in forty eyes

\begin{tabular}{|c|c|c|c|}
\hline \multicolumn{2}{|c|}{ Vortex veins occluded } & \multirow{3}{*}{$\begin{array}{l}\text { No. of } \\
\text { eyes } \\
-\end{array}$} & \\
\hline Number & Name & & \\
\hline \multirow[t]{2}{*}{ All } & - & & I 4 \\
\hline & $\overline{\mathrm{ST}+\mathrm{SN}+\mathrm{IT}}$ & 7 & \\
\hline \multirow[t]{2}{*}{3} & $\mathrm{ST}+\mathrm{IT}+\mathrm{IN}$ & I & 9 \\
\hline & $\mathrm{ST}+\mathrm{SN}+\mathrm{IN}$ & I & \\
\hline \multirow{3}{*}{2} & $\mathrm{ST}+\mathrm{SN}$ & 4 & \\
\hline & $\mathrm{ST}+\mathrm{IT}$ & 5 & 12 \\
\hline & $\mathrm{SN}+\mathrm{IT}$ & 3 & \\
\hline \multirow{3}{*}{ I } & $\overline{\mathrm{ST}}$ & 3 & \\
\hline & $\mathrm{SN}$ & I & 5 \\
\hline & IT & I & \\
\hline
\end{tabular}

Table II Follow-up periods

\begin{tabular}{|c|c|c|}
\hline $\begin{array}{l}\text { Number of } \\
\text { vortex veins } \\
\text { occluded }\end{array}$ & $\begin{array}{l}\text { No. of } \\
\text { eyes }\end{array}$ & $\begin{array}{l}\text { Follow-up } \\
\text { period } \\
\text { (days) }\end{array}$ \\
\hline \multirow{4}{*}{ All } & 8 & o \\
\hline & 2 & 85 \\
\hline & 2 & $9^{I}$ \\
\hline & 2 & $9^{8}$ \\
\hline \multirow{6}{*}{3} & 4 & o \\
\hline & I & 4 \\
\hline & I & 14 \\
\hline & I & 20 \\
\hline & I & 27 \\
\hline & I & 28 \\
\hline \multirow{4}{*}{2} & 6 & o \\
\hline & 3 & 4 \\
\hline & 2 & 20 \\
\hline & I & $2 \mathrm{I}$ \\
\hline \multirow{2}{*}{ I } & 4 & 0 \\
\hline & I & $2 \mathrm{I}$ \\
\hline
\end{tabular}

IN $=$ Inferior nasal

IT $=$ Inferior temporal

SN $=$ Superior nasal

$\mathrm{ST}=$ Superior temporal

(i) external ogular examination

This was performed in all the eyes, and included a detailed slit-lamp examination in most cases.

(2) FUNDUS EXAMINATION

This was mostly limited to the posterior part of the fundus because of the difficulty of examining the peripheral parts. In some of these, however, the equatorial part of the fundus was examined. Therefore the presence of fundus lesions in the equatorial part of the fundus in only a few eyes is to be judged in the light of this limitation, and does not represent the true incidence of such lesions.

(3) intravenous FLUORESGin FUndus ANGiOgRAPHY

This examination was again limited to the posterior part of the fundus in the majority of the eyes.

(4) intraogular PRessure (iop) estimation

This was measured with a Schiötz tonometer after the fundus examination and angiography to avoid interfering with the corneal transparency. Since a lid retractor was required to keep the eyes wide open for examinations 1,2 , and 3 above, the prolonged use and manipulation of the retractor caused pressure on the eyeball with a consequent fall in IOP. The IOP recorded may therefore be somewhat lower than the actual pressure in these eyes.

(5) Histopathological or Silicone RUBber PERfusion Examination

All the eyes were first perfused with gluteraldehyde via the common carotid arteries. Silicone rubber perfusion was carried out after this in thirteen eyes by a method already described (Hayreh and Baines, 1972a).

\section{Observations}

ogGlusion OF ALl the VORTEX VEINSin the Eye (i 4 eyes)

Anterior chamber $(A C)$

A hyphaema appeared in all the eyes, except four belonging to animals which were killed 
less than half an hour after the occlusion. The start of the bleeding varied from a few minutes to about 3 hours after the occlusion. It came from the posterior chamber via the pupil and usually developed gradually to a total hyphaema which cleared in 2 to 5 weeks.

Slit-lamp examination in some of these eyes, before the hyphaema developed, showed a proteinous, gelatinous material filling the AC. On injection of intravenous fluorescein, a profuse leakage of fluorescein from the posterior chamber via the pupil into the AC was seen in all the eyes.

The AG was shallow immediately after occlusion because of the pushing forward of the iris-lens diaphragm (Fig. I a). On follow-up of six eyes, no AC was seen in three when the hyphaema had cleared, because the iris was in contact with the posterior surface of the cornea; however, a very shallow AG was still seen in the pupillary region (Fig. I $b$ ). In the other three eyes, the AC was either of normal depth or possibly slightly shallower than normal.
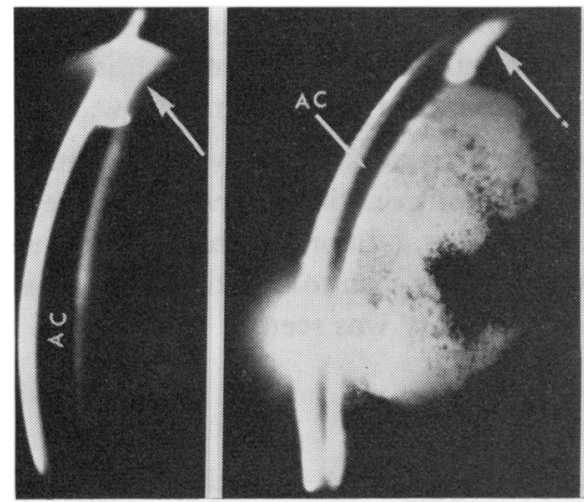

(a)

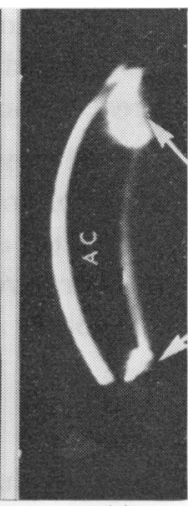

(c)

FIG. I Slit-lamp photographs of anterior segment of eyes, with occlusion of VVs (Arrows indicate iris)

(a) About 15 min. after occlusion o all the VVs, showing a very shallow anterior chamber (present only in the pupillary region).

(b) 9 I days after occlusion of all VVs, showing a very shallow anterior chamber (present only in the pupillary region) and cataractous lens.

(c) One week after occlusion of three VVs, showing a shallow anterior chamber.

\section{Cornea}

In two of the eyes, in which the AC was absent, corneal stromal vascularization was seen to extend from the limbus towards the centre by radial vessels, with the central part usually not involved. Similar corneal vascularization was also seen in another eye with a normal AG.

\section{Iris}

The iris was hyperaemic soon after the occlusion. With the hyphaema it was not possible to assess the early changes in the iris. In three of the six eyes followed-up for 3 months, iris atrophy (involving half of the iris in two and the entire iris in one) was seen, with complete or partial anterior and posterior synechiae. In two of these three eyes, rubeosis of the iris was also seen.

\section{Lens}

Soon after the occlusion of the veins, the anterior lens surface was covered with proteinous material and/or blood. Later, a variable amount of dark brown pigmentary deposit was seen on the anterior surface in the pupillary region in almost all eyes and in one eye mainly on its posterior surface. In three eyes with posterior synechiae and absent AG, the lens became completely cataractous in less than 5 weeks (Fig. I $b$ ). 
Intraocular pressure (IOP)

Immediately after the occlusion of the VVs, the IOP was invariably elevated, usually to about $40-60 \mathrm{~mm}$. $\mathrm{Hg}$. This rise in the IOP was not related to the presence of hyphaema. On the next day, the IOP was as high as or higher than the immediate postocclusion pressure. The raised IOP was associated with corneal epithelial oedema. Usually the IOP returned to near normal in about a week. This fall in IOP was progressive and reached its lowest recordable level 9 to 10 days after the occlusion in eyes in which the AC was still present, but about 2 weeks after occlusion in eyes in which it was absent. After this period, the IOP slowly recovered in 5 to 6 weeks if the AC was present, although not quite to the normal level. If the $\mathrm{AG}$ was absent, there was permanent marked hypotony with unrecordable pressure.

\section{Fundus}

In eight out of the fourteen eyes (i.e. those without hyphaema), post occlusion examination of the fundus was possible. It showed no ophthalmoscopic abnormality in the posterior part. In the remaining six eyes, an early hyphaema obscured the view.

In six eyes followed for 85 to 98 days, fundus examination was possible in only three, with a hazy view in one (due to pigment deposit on the posterior surface). In the remaining three, the lens was completely cataractous. In one of the eyes with a clear view of the fundus, a chorio-retinal degenerative patch was seen to be situated just posterior to the equator at about 6 o'clock. No other abnormality was seen.

\section{Fluorescence fundus angiography}

This was performed soon after the occlusion in eight eyes with clear media. In a normal rhesus monkey eye the choroidal vascular bed starts to fill before the retinal arteries, and the choroidal filling is complete by the retinal arterial or early arterio-venous phase. However, in these eyes with all the VVs occluded, the choroid did not start to fill until the retinal arterio-venous phase, or even much later (Fig. 2a, b). The choroidal filling was very sluggish, so that the choroid had not filled completely even by the end of the retinal-venous phase. The temporal part of the choroid usually filled earlier than the nasal part (Fig. 2c). The watershed areas between the distribution of the various VVs were the last to fill (Fig. 2c). Frequently the region of the choroid drained by one of the veins filled earlier or later than the regions of other VVs.

On follow-up, in three eyes (two followed up for 85 and one for 98 days), in which fundus examination was possible at the termination of the experiment, fluorescence angiography revealed that the choroidal circulation was restored to normal (Fig. 3), with a complete filling of the choroid by the early arterio-venous phase. In the eye with chorio-retinal lesions, destruction of the choroid and the pigment epithelium in the lesion was seen. No other abnormality was detected.

OGGLUSION OF THREE VORTEX VEINS IN THE EYE (in 9 eyes)

\section{Anterior chamber}

Bleeding from the posterior chamber into the AC was seen in four of five eyes followed-up for 24 hours or longer. This usually started within about an hour of the occlusion (in 
(a)

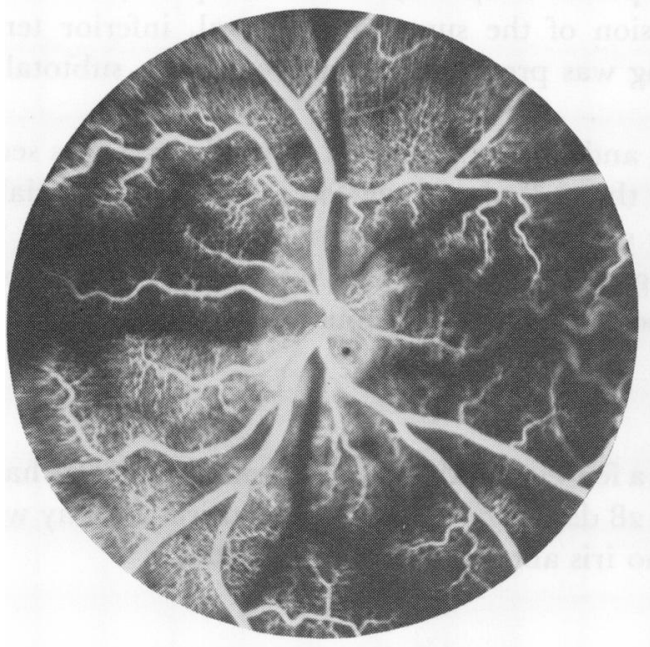

(c)
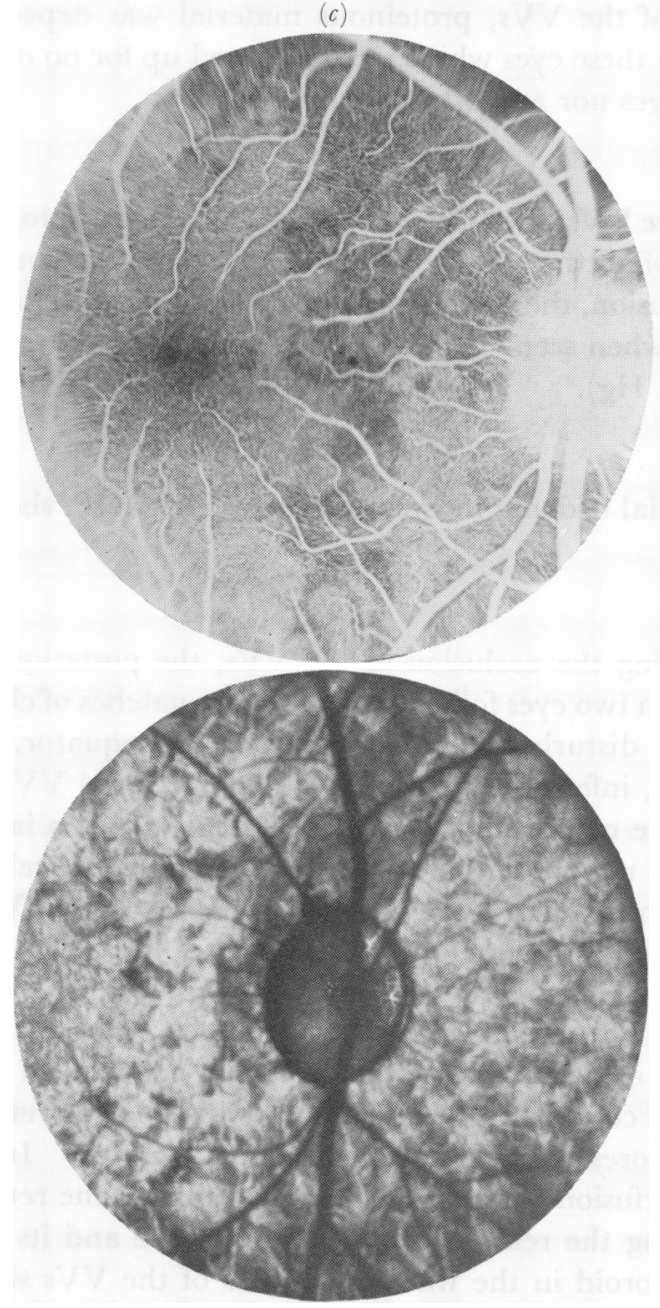

(b)

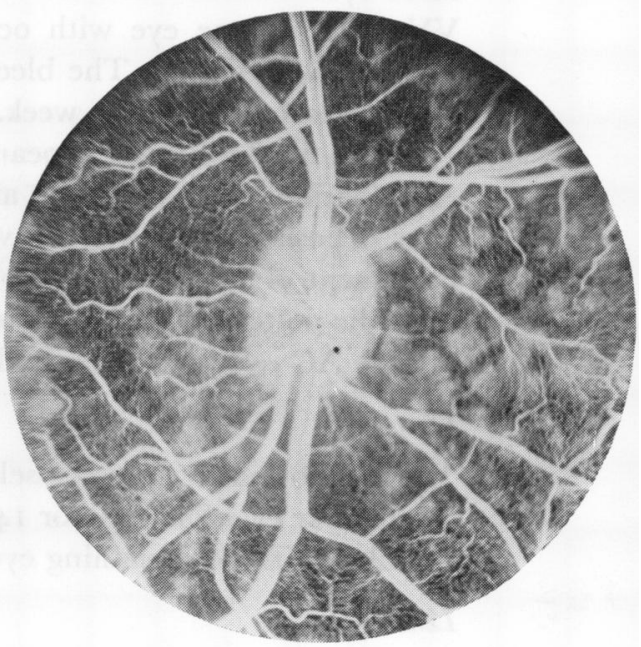

FIG. 2 Fluorescence fundus angiograms of right eye I hour after occlusion of all VVs

(a) During retinal arterial phase, showing complete absence of choroidal filling.

(b) During late retinal arterio-venous phase, showing start of choroidal filling.

(c) During retinal post-venous phase, showing areas of filled and unfilled choroid.

Figs $\mathrm{x} b$ and $\mathrm{s} o b$ show changes in the anterior segment 9I days after occlusion in the same eye.

FIG. 3 Fluorescence fundus angiogram of right eye 98 days after occlusion of all VVs during preretinal-arterial phase, showing normal choroidal filling

Fig. Io $a$ shows changes in the anterior segment in the same eye. 
three eyes with occlusion of the superior temporal, inferior temporal, and superior nasal VVs, and in one eye with occlusion of the superior temporal, inferior temporal, and inferior nasal VVs). The bleeding was progressive and produced a subtotal hyphaema, which cleared in about a week.

Before the hyphaema appeared, and in eyes in which no hyphaema was seen, slit-lamp examination of the $\mathrm{AC}$, soon after the occlusion, revealed proteinous material and gelatinous deposits. The AC was very shallow, with a narrow angle due to the forward displacement of the iris-lens diaphragm (Fig. Ic). There was a marked leak of fluorescein from the posterior chamber into the AC after intravenous injection of the dye. On followup, the AC returned to normal.

Iris

The iris showed engorged vessels in a few eyes and in one even small punctate haemorrhages. Of the four eyes followed for 14 to 28 days, one showed sectoral iris atrophy with posterior synechiae; in the remaining eyes no iris abnormality was detected.

Lens

Immediately after the occlusion of the VVs, proteinous material was deposited on the anterior lens surface. The lens, in these eyes which were followed up for no more than 28 days, showed no cataractous changes nor any other abnormality.

\section{Intraocular pressure}

Immediately after occlusion of three VVs, the IOP was elevated to about 30 to $40 \mathrm{~mm}$. $\mathrm{Hg}$, but not so high as after occlusion of all the VVs. The raised IOP was also present on the next day, but a week after the occlusion, the eye had gone very soft. A week later the IOP had started to recover slowly but when seen 3 to 4 weeks after the occlusion, the eyes still had a low IOP (about IO-I $5 \mathrm{~mm}$. $\mathrm{Hg}$ ).

\section{Cornea}

The cornea, except for the epithelial oedema in eyes with very high IOP, showed no abnormality.

\section{Fundus}

In the period immediately following the occlusion of the VVs, the posterior part of the fundus showed no abnormality. In two eyes followed for 28 days, patches of chorio-retinal lesions, with granular pigmentary disturbance, were seen near the equator. In one of these eyes (with superior temporal, inferior temporal, and superior nasal VVs occluded), these lesions were distributed in the regions of the three occluded veins - a large number of patches in the superior temporal region, and fewest in the inferior temporal sector. In the second eye (with superior temporal, inferior temporal, and inferior nasal VVs occluded) a rounded patch was seen on the temporal side.

\section{Fluorescence fundus angiography}

Soon after occlusion, the quadrant of the choroid supplied by the unoccluded VV was the first to show fluorescence and filled completely during the early part of the retinal arteriovenous phase (Fig. 4a, b). This fluorescent area had well-defined margins. In the regions of the occluded VVs, very slow perfusion of the choroid started during the retinal arterial phase; perfusion was patchy during the retinal arterio-venous phase and its distribution tended to be sectoral, with the choroid in the watershed areas of the VVs showing very 


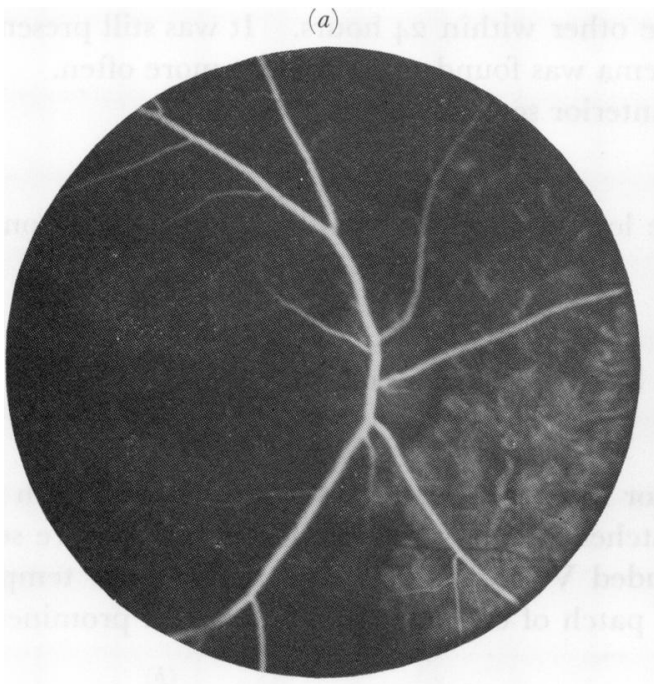

(c)

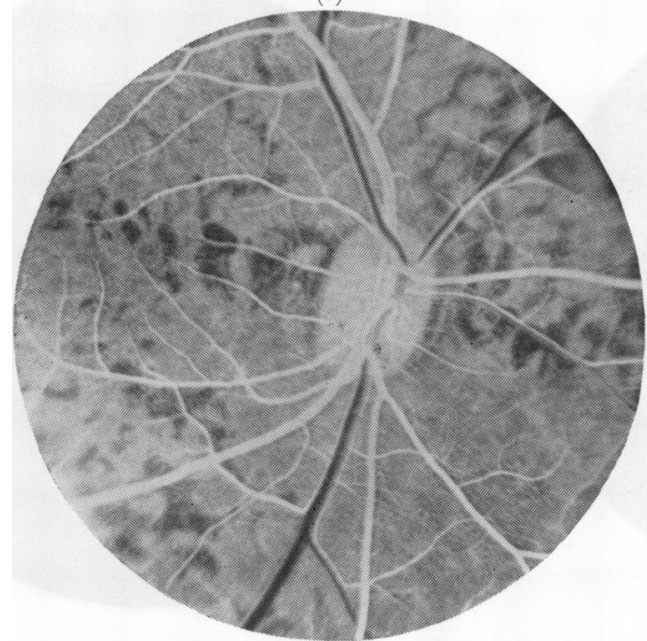

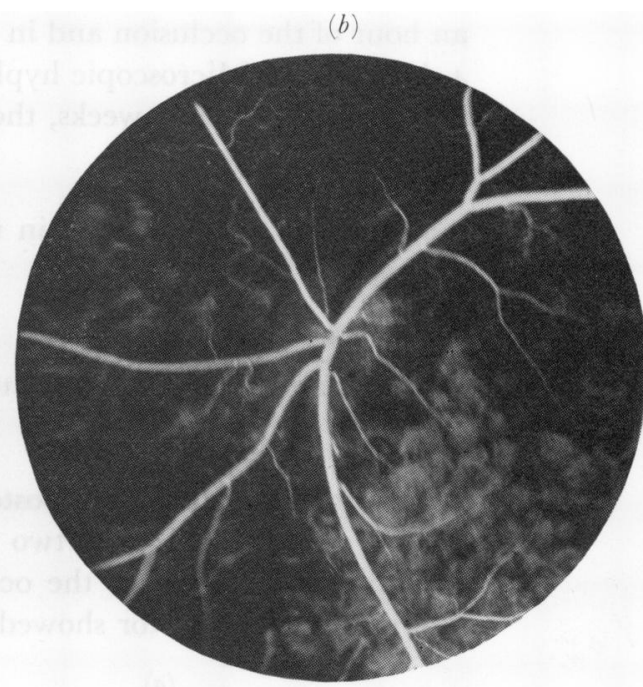

FIG. 4. Fluorescence fundus angiograms after occlusion of $3 \mathrm{VVs}$

(a) Right eye half an hour after occlusion of superior temporal, superior nasal, and inferior temporal VVs, during retinal arterial phase, showing normal filling of only inferior nasal quadrant of the choroid.

(b) Left eye 2 hours after occlusion of superior temporal, superior nasal, and inferior nasal VVs during retinal arterial phase, showing normal filling of only inferior temporal quadrant of the choroid.

(c) Right eye I hour after occlusion of superior nasal, and inferior temporal VVs during retinal arterio-venous phase, showing unfilled watershed zones and complete filling of inferior nasal quadrant of choroid, with incomplete choroidal filling in other three quadrants.

poor filling or none (Fig. 4c). Complete filling of the choroid took place only by the late venous phase - the watershed areas and adjacent parts of the peripapillary choroid were the last to fill. By this time the first normally filled sector of the choroid of the unoccluded VV had started to empty. No abnormality in the retinal circulation was observed.

On follow-up, the choroidal circulation improved nearly to normal within 2 weeks, so that complete filling of the choroid was seen by the retinal arterio-venous phase.

OGGLUSION OF TWO VORTEX VEINS IN THE EYE (in I 2 eyes)

Anterior chamber

In these eyes, the major and constant abnormality detected in the anterior segment was the deposition of proteinous material on the anterior lens surface and its accumulation in the $\mathrm{AC}$ in about an hour. This produced a gelatinous deposit in the dependent part of the AC. On fluorescence angiography, a marked leak of fluorescein from the posterior chamber in the sector of the occluded veins was seen, which at first produced a localised fluorescence of the involved region. A frank hyphaema was seen in two eyes: in one within 
an hour of the occlusion and in the other within 24 hours. It was still present when seen 4 days later. Microscopic hyphaema was found to be present more often.

On follow-up for 3 weeks, the anterior segment became normal.

Lens

No abnormality was found in the lens apart from the proteinous deposit on its anterior surface.

\section{Intraocular pressure}

This was not significantly affected by such an occlusion.

\section{Fundus}

No lesion was seen in the posterior fundus immediately after the occlusion of the VVs. After 3 weeks, in one eye, two patches of chorio-retinal degeneration were seen near the equator, in the areas of the occluded VVs (superior nasal and inferior temporal). The inferior temporal sector showed a patch of chorio-retinal lesion and prominent choroidal
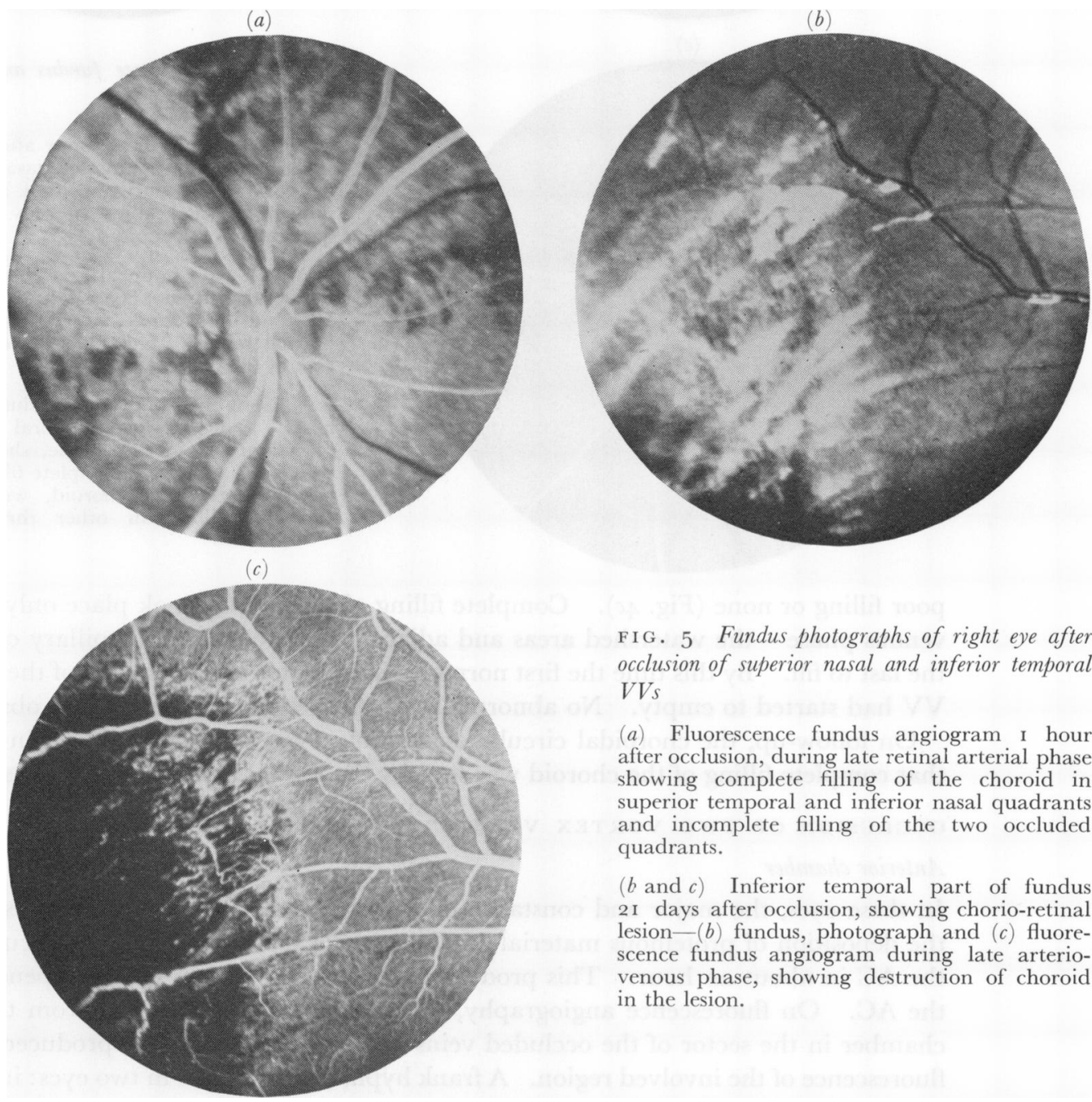

FIG. 5 Fundus photographs of right eye after occlusion of superior nasal and inferior temporal $V V_{S}$

(a) Fluorescence fundus angiogram I hour after occlusion, during late retinal arterial phase showing complete filling of the choroid in superior temporal and inferior nasal quadrants and incomplete filling of the two occluded quadrants.

( $b$ and $c$ ) Inferior temporal part of fundus 2 I days after occlusion, showing chorio-retinal lesion- $(b)$ fundus, photograph and $(c)$ fluorescence fundus angiogram during late arteriovenous phase, showing destruction of choroid in the lesion. 
vessels and whitish linear lesions with choroidal atrophy (Fig. $5^{b}$ ). The superior nasal region showed a small, pale, rounded patch of degeneration.

\section{Fluorescence fundus angiography}

Soon after the occlusion of the VVs, a very sluggish and poor choroidal circulation was seen in the areas of the occluded veins, but a normal choroidal circulation in the regions of the unoccluded ones (Figs 5a, 6a,c). Each sector had well-defined margins. The normal areas filled uniformly during either the retinal arterial or the early arterio-venous phase (Figs $5 a, 6 a, c)$. The choroid in the region of the occluded VVs did not fill properly till the late retinal arterio-venous phase, leaving well-defined empty watershed zones between the occluded and unoccluded VVs (Fig. 6b), which did not fill completely till the late retinal-venous phase; thus a complete filling occurred by the late venous phase.

On follow-up of these eyes, the choroidal circulation recovered to normal after about a week. In the area of the chorio-retinal degenerative patch near the equator, almost complete destruction of the choroid was seen, only a few choroidal vessels being present (Fig. $5^{c}$ ). Some choroidal unmasking was seen at the junctional zones between the normal and atrophic areas.
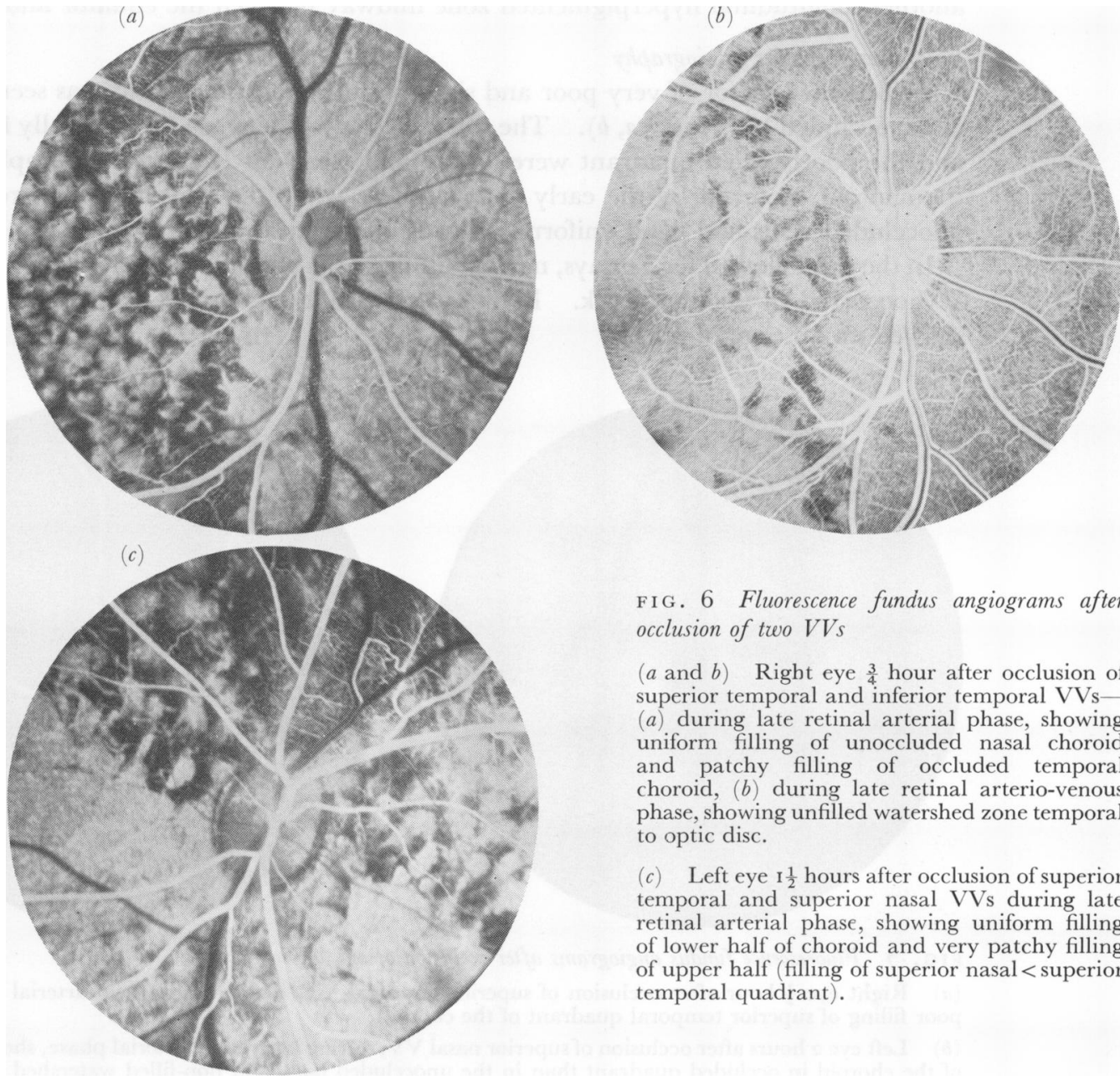

FIG. 6 Fluorescence fundus angiograms after occlusion of two VVs

$\left(a\right.$ and $b$ ) Right eye $\frac{3}{4}$ hour after occlusion of superior temporal and inferior temporal VVs(a) during late retinal arterial phase, showing uniform filling of unoccluded nasal choroid and patchy filling of occluded temporal choroid, $(b)$ during late retinal arterio-venous phase, showing unfilled watershed zone temporal to optic disc.

(c) Left eye $\mathrm{I} \frac{1}{2}$ hours after occlusion of superior temporal and superior nasal VVs during late retinal arterial phase, showing uniform filling of lower half of choroid and very patchy filling of upper half (filling of superior nasal < superior temporal quadrant). 
OCGLUSION OF ONE VORTEX VEIN IN THE EYE (in 5 eyes)

Anterior Chamber

The changes in the anterior segment were identical to those seen after occlusion of two veins. The only constant abnormality was the presence of proteinous material on the anterior surface of the lens and in the AC within $\frac{1}{2}$ to $\mathrm{I}$ hour after the occlusion and a gelatinous deposit in the AC. On injection of fluorescein intravenously, a localized fluorescein leak from the posterior chamber into the $\mathrm{AC}$ was seen in the occluded sector only.

Intraocular pressure

This was normal in all the eyes.

Lens

No abnormality was found apart from the proteinous deposit on its anterior surface.

Fundus

Examination of the posterior fundus, soon after the occlusion, revealed no abnormality. In an eye followed for $2 \mathrm{I}$ days after the occlusion of the superior temporal $\mathrm{VV}$, the superior temporal sector showed a rounded chorio-retinal degenerative lesion near the equator and another longitudinal hyperpigmented zone midway between the equator and macula.

\section{Fluorescence fundus angiography}

A well-defined sector of very poor and sluggish filling of the choroid was seen in the area of the occluded VV (Fig. $7 a, b)$. The watershed zones between the normally filled choroid and the poorly filled quadrant were empty and clear-cut (Fig. $7 b$ ). Complete filling of the choroid occurred by the early venous phase while the choroid in the regions of the unoccluded veins had filled uniformly during the arterial phase (Fig. 7).

In the eye followed for $2 \mathrm{I}$ days, normal filling of the choroid in the region of the occluded VV occurred in about a week. In the area with the chorio-retinal lesion, the choroid showed an absence of filling.

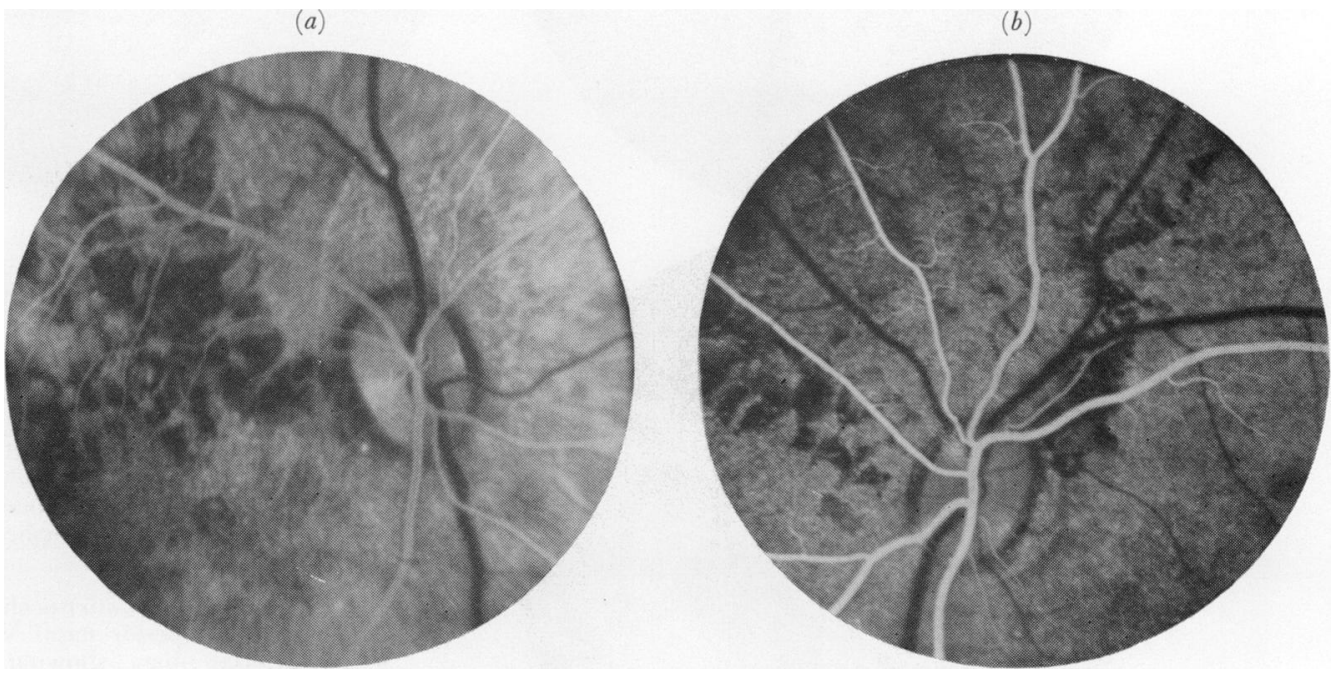

FIG. 7 Fluorescence fundus angiograms after occlusion of one VV

(a) Right eye $\frac{1}{2}$ hour after occlusion of superior temporal $\mathrm{VV}$, during late retinal arterial phase, showing poor filling of superior temporal quadrant of the choroid.

(b) Left eye 2 hours after occlusion of superior nasal VV, during late retinal arterial phase, showing less filling of the choroid in occluded quadrant than in the unoccluded part, and non-filled watershed zones. 


\section{Discussion}

The arterial blood supply to the uveal vascular bed takes place via the 2-3 posterior ciliary arteries and about 8-10 anterior ciliary arteries. The venous drainage from the uveal tract is carried almost entirely by the 3-4 VVs in rhesus monkeys (Hayreh, 1964). It is generally assumed that the arteries, veins, and chorio-capillaris in the choroid and the rest of the uveal tract communicate freely. Our previous studies have shown that the posterior ciliary arteries have a segmental supply in the choroid (Hayreh, 1970, I97 I ; Hayreh and Baines, 1972a); there was also evidence that the chorio-capillaris did not form a freely communicating vascular bed but was composed of tiny independent zones, arranged in a form of a mosaic, and the adjacent zones showed no evidence of communication with one another. Interestingly enough, the VVs have also revealed segmental distribution in the choroid, as judged from our present study. The arterial and venous segments in the choroid do not have exactly the same distribution.

The following is a detailed description of the pathological changes seen in the various ocular tissues following occlusion of the various VVs.

\section{GHOROIDAL CIRGULATION}

On intravenous fluorescein fundus angiography, the choroidal vascular bed normally starts to fill during the pre-retinal arterial phase and the filling is complete by the retinal arterial or early arterio-venous phase. When all the VVs in an eye were occluded, however, the choroidal bed showed no filling at all until the arterio-venous phase (Fig. 2). Even the choroidal perfusion was extremely sluggish and patchy, so that complete filling of the choroidal bed did not occur even by the end of the retinal venous phase; the temporal part of the choroid filled earlier than the nasal part, and the territory of one VV could fill before that of another. This delay in the filling of the choroidal vascular bed is primarily due to back pressure in the uveal vascular bed as a result of complete blockage of its outlet; the raised IOP is also to some extent a contributory factor (Hayreh, I969; Hayreh, Revie, and Edwards, I970).

On occluding three VVs, angiography showed normal filling of a well-defined quadrant of the choroid corresponding to the unoccluded VV (Fig. $4 a, b$ ). The choroid drained by the occluded VVs started to fill slowly during the late retinal arterial phase and the filling was not complete till the late retinal venous phase. Similarly, eyes with occlusion of one or two VVs (Figs $5 a, 6 a, c, 7$ ) showed in the choroid either a well-defined quadrantic normal filling, or a very sluggish filling, according to whether the VV was unoccluded or occluded. The watershed zones between the various VVs were well-defined and were the last to fill (Figs 2c, 4c,6b, $7 b$ ). The fact that the chorio-capillaris in the watershed zones did not fill until very late, while the chorio-capillaris on either side (Figs 2c, 4c, 6b, $7 b$ ) filled completely, further confirms the segmental nature of the chorio-capillaris. Thus, each VV has a well-defined segmental distribution in the choroid, with little communication with the adjacent vein. A similar sectoral distribution of the VVs has also been recorded by other workers in rabbits and dogs (Leber, 1873; Koster, 1895; Sachsenweger and Lukoff, I959).

The watershed zone between the upper and lower VVs in the eyes of rhesus monkeys mostly passed horizontally through the macula and optic disc region (Figs $4 c, 5 a, 6 c$, $7 a$ ), while that between the temporal and nasal veins in the majority passed vertically either slightly temporal to the peripapillary region or through the temporal peripapillary choroid (Figs 2c, 5a,6b,c,7). Sometimes the choroidal territory of the various VVs may 
not be of the same size and also not completely quadrantic, depending upon the size and number of VVs in an eye.

On follow-up, the choroidal perfusion in the region of the occluded VVs was restored to normal in I week after the occlusion of one or two VVs, and in 2 weeks after the occlusion of three veins. It was not possible to determine the time taken for such a restoration of choroidal perfusion after occlusion of all the VVs because hyphaema lasted for a few weeks and later on there were cataractous changes in half of the cases; however, it was normal when seen after about 3 months (Fig. 3). The exact mode of this restoration of the VV outflow is not known. Koster (1895), in his studies of occlusion of all the VVs in rabbits, found after injection of Berlin blue gelatine, that the occluded VVs established small collateral vessels at their point of exit from the sclera; the collateral vessels went forwards and backwards to the adjacent muscles. He also recorded the presence of enlarged collateral vessels between the choroidal and retinal vessels around the optic nerve, but did not find any collaterals between the ciliary body and the overlying episcleral plexus or muscles. Our silicone rubber perfusion studies have not been conclusive in this respect, except that in some of the eyes, after silicone injection, the normal stem of the VV outside the globe was seen to have re-formed in spite of its having been cut with a cautery at the time of occlusion. We found no other collateral of any significance between the VVs and the extraocular venous channels.

\section{FUNDUS}

Immediately after occlusion of the VVs no lesions were seen in the posterior part of thefundus. The equatorial and peripheral parts of the fundus could not usually be properly examined.

Histological studies immediately after the occlusion have shown a markedly engorged choroidal bed and even choroidal and suprachoroidal haemorrhages in the region of the occluded VVs (Fig. 8).

On follow-up, patches of chorio-retinal atrophy were seen near the equator in the region of the occluded VV (in one eye each with occlusion of four, two, and one VV, and two eyes with three VVs occluded) (Fig. $\left.5^{b}, c\right)$. In some of the remaining eyes such lesions could have been missed because of the difficulty in examining the peripheral part of the fundus in these eyes. On angiography, these lesions show marked destruction of the choroidal vascular bed (Fig. $5^{c}$ ). We feel that the chorio-retinal lesions are secondary to the choroidal haemorrhages seen in these eyes.

Sachsenweger and Lukoff (1959) described the occurrence of a flat, circumscribed or mould-like retinal detachment after the occlusion of two or three VVs in dogs and a total retinal detachment after the occlusion of four VVs. The latter group also had intrachoroidal and subretinal haemorrhage. Koster (1895) found histological evidence of retinal detachment in some eyes after occlusion of two to four VVs in rabbits and a total detachment 4 months after occlusion of four VVs. All of these workers reported the presence of subretinal transudate in their retinal detachments which was considered to be due to venous congestion by Sachsenweger and Lukoff (1959). Similarly, de Faria and Silva (1950) thought that extensive retinal detachment with choroidal tumours in the posterior part of the choroid were secondary to compression of the VVs and associated transudation. Cogan ( 1960 ) explained bilateral retinal detachment in a case with carotid cavernous fistula on the same basis. Klien (I96I) suggested that central serous retinopathy was due to stasis in the VVs, because on histopathological examination of three eyes with central serous retinopathy she found congestion and stasis in the VV bed and also in the other orbital and retinal venous beds. Recent fluorescein fundus angio- 


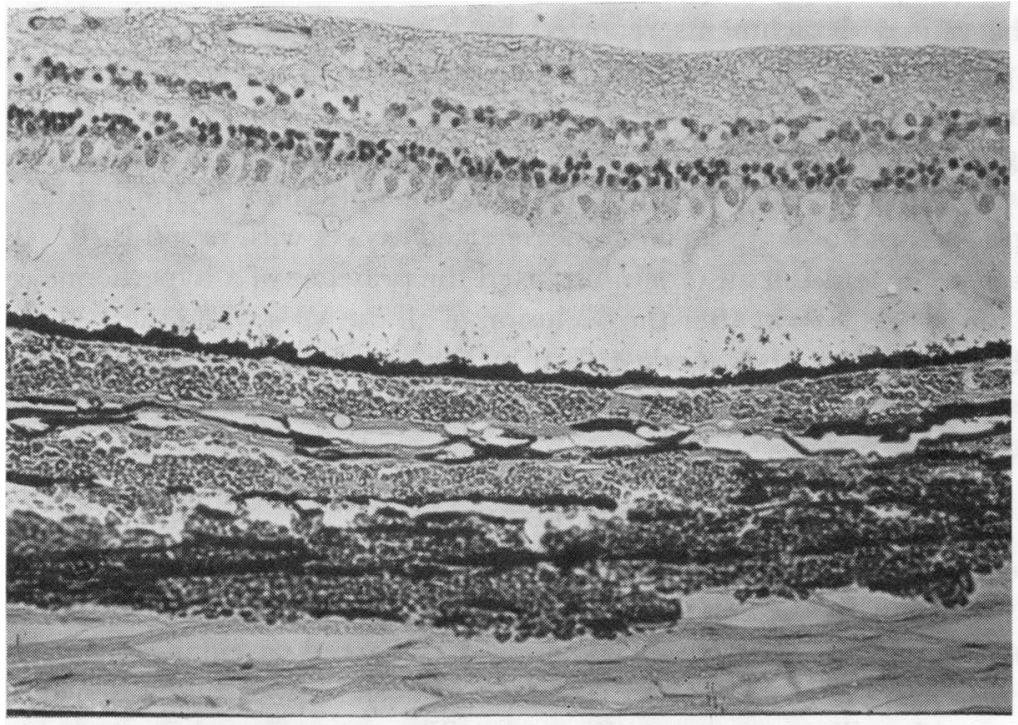

(a)

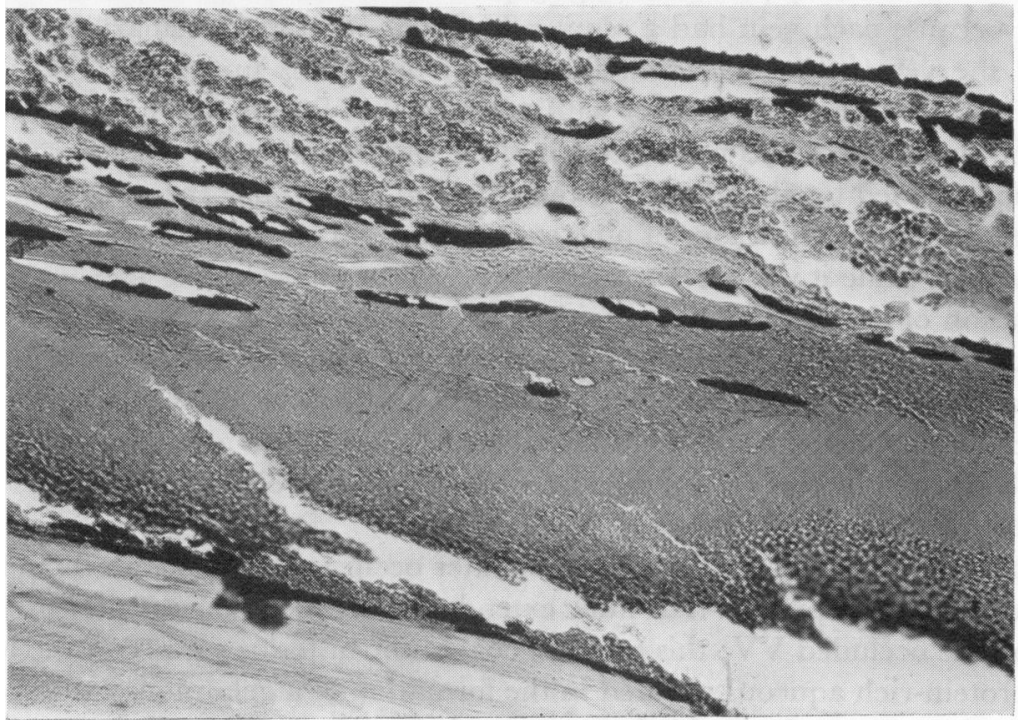

(b)

FIG. 8 Photomicrographs of eyes $\mathrm{I}$ to 2 hours after occlusion of all VVs, showing

(a) choroidal haemorrhages.

(b) choroidal and suprachoroidal haemorrhages.

Retinal detchment in $(a)$ is an artefact. Haematoxylin and eosin. $\times 3^{10}$

graphic studies of the eyes of patients with central serous retinopathy have lent no support to Klien's view. In our studies, no retinal detachment was seen ophthalmoscopically in any of our animals; nor did histology reveal any retinal detachment and associated subretinal transudation. Where all the VVs in the eye were occluded and the eye had become markedly hypotonic and degenerate with a cataract, histological examination revealed retinal detachment; such a detachment was considered to be part of a generalized degeneration and disorganization of the eye. Out of the remaining eyes, if a retinal detachment was detected histologically, it was considered to be an artefact and not a true detachment and there was no associated subretinal transudation. We feel that 
most of the retinal detachments reported by Koster (1895) were histological artefacts.

In the present study no clinical or histological evidence of choroidal detachment was seen in any eye.

Histological examination of some discs with all or three VVs occluded showed in a few cases some evidence of cupping and atrophy of the optic disc. Ophthalmoscopically, no definite optic atrophyor cupping could be detected in eyes with raised IOP. Koster (1895) and Sachsenweger and Lukoff (I959) reported the presence of a hyperaemic optic disc and distended retinal veins soon after the occlusion of all the VVs when the IOP was very high. We feel that the fundus changes described by these authors were secondary to the marked ocular hypertension. Koster (1895) found these discs to become pale eventually, with gliosis of the optic nerve.

\section{GILIARY PROGESSES AND GILIARY BODY}

In the present study, the main brunt of this uveal venous congestion induced by the VV occlusion was borne by the vessels in the ciliary processes. Histological examination of the eyes, soon after the occlusion of the VVs, revealed marked vascular congestion and dilated vessels in the ciliary processes (Fig. 9); some eyes even showed the presence of thin-walled blood cysts on the ciliary processes (Fig. 9a). The area affected depended upon the VV occluded, because each vein had a segmental distribution in the ciliary processes as well. When all the veins were occluded, the fine blood vessels under the ciliary epithelium ruptured in the majority of the eyes within a period of from a few minutes up to about 3 hours after occlusion. This produced bleeding into the posterior chamber which escaped via the pupil and led to a hyphaema in the AG (Fig. 9a). When three VVs were occluded, such a bleeding was seen in four of nine eyes, (or in four of five eyes followed-up for more than 24 hours), while it was seen in two of twelve eyes on occluding two VVs, but in none after occlusion of one VV. In eyes with occlusion of three and two VVs, the hyphaema was not only less frequent but also less marked than after occlusion of four VVs. The hyphaema cleared in the former eyes in a week and in the latter in 2 to 5 weeks. The occurrence of hyphaema with occlusion of all the VVs was also seen by Leber (1873), Weber (1877), and Koster (1895) in rabbits, but none was observed by Sachsenweger and Lukoff (1959) in dogs.

In all the eyes of this series, immediately after occlusion, and before the appearance of any hyphaema, thick protein-rich fluid extended from the ciliary processes in the sector drained by the occluded VV; this exudate coated the anterior surface of the lens. In the $\mathrm{AC}$, the protein-rich aqueous resulted in the formation of a gelatinous cobweb-like deposit in its dependent part. It would thus appear that a marked rise in blood pressure in the capillaries of the ciliary processes results in the outpouring of protein-rich aqueous. Histological examination of some of these eyes revealed ciliary epithelial cysts projecting into the posterior chamber (Fig. $9 b$ ). Possibly sluggish circulation, by producing defective oxygenation of the ciliary epithelium, could be another contributory factor in the breakdown of the blood-aqueous barrier, resulting in the production of protein-rich aqueous. Soon after the injection of intravenous fluorescein, profuse leakage of fluorescein from the involved sector only was seen; this was at first localized to the involved sector of the pupil and AC but progressively became more prominent and diffuse (Fig. I I). The blood, protein-rich aqueous, and fluorescein from the posterior chamber passed through the pupil on to the anterior lens surface and $A G$, but none was seen in the vitreous or on the posterior lens surface. The vitreous in all eyes was clear. On follow-up, the protein-rich aqueous cleared with the return to normal of the uveal circulation. 

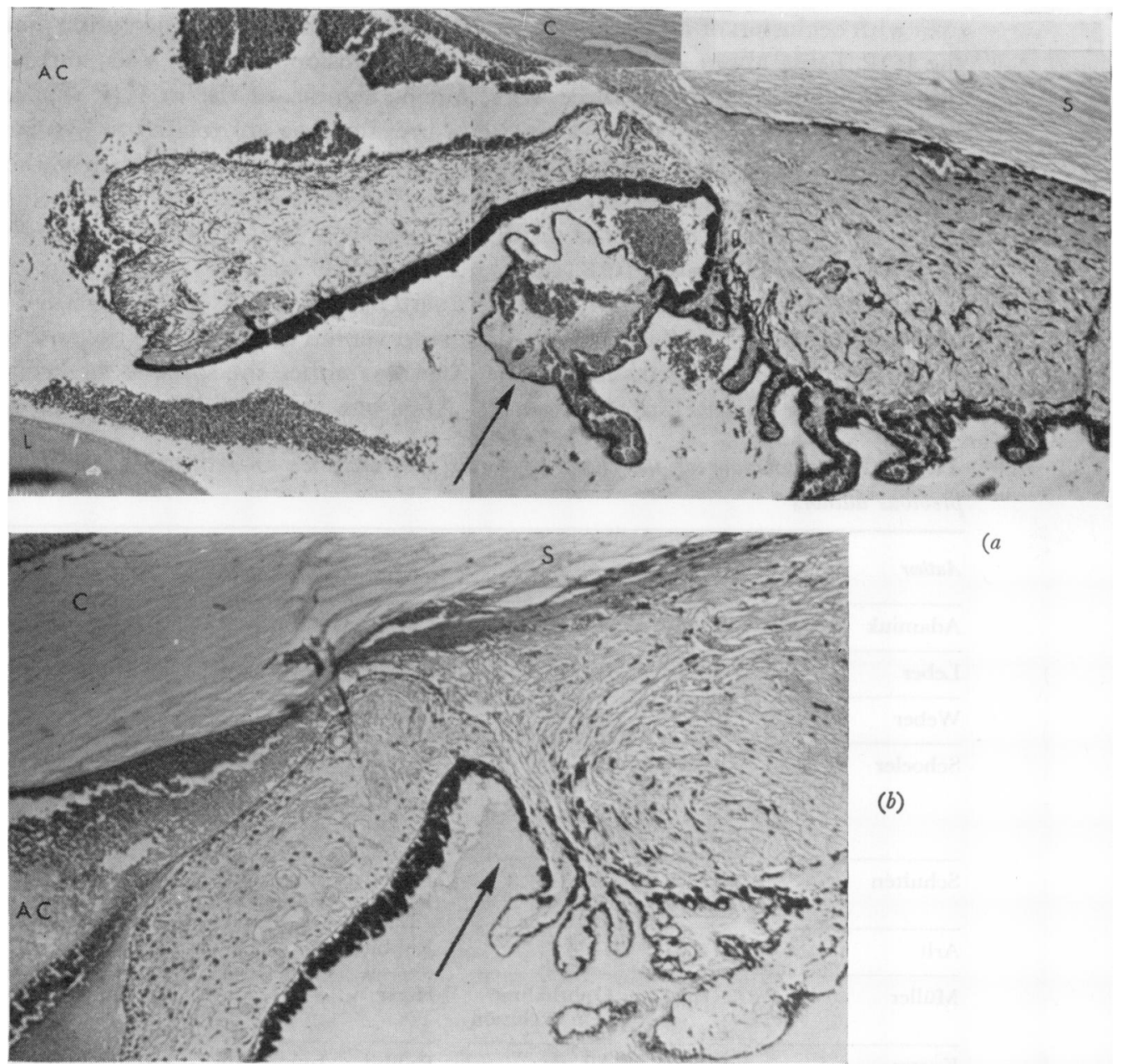

$(a$

FIG. 9 Photomicrographs of anterior segment of eyes i to 2 hours after occlusion of all VVs

(a) Markedly engorged vessels in ciliary processes, a blood cyst (arrow), and haemorrhages in anterior and posterior chambers and pupil.

Fig. $8 a$ shows the same eye. Haematoxylin and eosin. $\times 95$

(b) Engorged vessels in ciliary body and iris, proteinous fluid in the posterior chamber (arrow), and a hyphaema in the anterior chamber.

Fig. $8 b$ shows the same eye. Haematoxylin and eosin. $\times 123$

DEPTH OF ANTERIOR GHAMBER

The iris-lens diaphragm moved forward significantly in eyes with occlusion of all or three VVs, because there was an increase in the volume of the posterior compartment of the eye, following marked venous congestion in the choroidal vascular bed. This resulted in a shallow AC - shallower after occlusion of all the VVs than after three veins (Fig. I). On follow-up, in three of six eyes with all the VVs occluded, the AC was found to be absent after the hyphaema had cleared and it never re-formed, while in the remaining three eyes the depth of the AC became nearly normal after the hyphaema had cleared. In eyes with occlusion of two or one VV, no significant change in the depth of the AC was noticed. The findings of Koster (1895) were similar.

INTRAOGULAR PRESSURE

The findings of previous workers are summarized in Table III. In the present series, 
eyes with occlusion of all or three VVs showed immediately after occlusion a marked rise in the IOP, being about 40 to $60 \mathrm{~mm}$. $\mathrm{Hg}$ after occlusion of all the VVs, and about 30 to 40 $\mathrm{mm}$. Hg after occlusion of three VVs; but no significant rise in IOP was recorded after occlusion of two or one VV. This rise in pressure was not related to hyphaema, because the IOP rose as much in eyes without as those with hyphaema. Similarly, the protein-rich aqueous seemed to play no role in the rise in IOP because the eyes with occlusion of one or two VVs had a large amount of gelatinous deposit in the AC without any rise in the IOP. In the eyes with elevated IOP, the angle of the AC was very narrow, and it could have been blocked in most of them by a forward shift in the iris-lens diaphragm and engorgement of the ciliary body and iris, because of marked venous congestion in the entire uveal tissue.

On the day after the occlusion, the IOP was either the same as or even higher than the immediate postocclusion pressure. After one week a fall in the IOP had set in, so

Table III Summary of the effects of vortex vein occlusion on intraocular pressure as observed by previous authors

\begin{tabular}{|c|c|c|c|c|c|}
\hline Author & Date & $\begin{array}{l}\text { No. of VVs } \\
\text { occluded }\end{array}$ & $\begin{array}{l}\text { Animal } \\
\text { used }\end{array}$ & $\begin{array}{l}\text { Time since } \\
\text { occlusion }\end{array}$ & Effect on intraocular pressure \\
\hline Adamiuk & $(\mathbf{1 8 6 7 )}$ & All & Cat & Immediate & up to $90 \mathrm{~mm} . \mathrm{Hg}$ \\
\hline Leber & $(1873)$ & All & Rabbit & Immediate & $4^{\mathrm{I}-5^{\mathrm{I}} \mathrm{mm} . \mathrm{Hg}}$ \\
\hline Weber & $(1877)$ & All & Rabbit & Immediate & Rapid rise \\
\hline Schoeler & $(1879)$ & All & Rabbit & Immediate & $\begin{array}{l}\text { Ligation of } \mathrm{VVs} \rightarrow 2 \mathrm{~mm} \text {. rise } \\
\text { Cauterization of } \mathrm{VVs} \rightarrow 18 \mathrm{~mm} \text {. } \\
\text { rise. } \\
\text { Soon returned to normal }\end{array}$ \\
\hline Schultén & $(1884)$ & $\begin{array}{l}\text { All } \\
2\end{array}$ & $\begin{array}{l}\text { Rabbit } \\
\text { Rabbit }\end{array}$ & $\begin{array}{l}\text { Immediate } \\
\text { Immediate }\end{array}$ & $\begin{array}{l}6 o-80 \mathrm{~mm} . \mathrm{Hg} \\
27-52 \mathrm{~mm} . \mathrm{Hg}\end{array}$ \\
\hline Arlt & ( 1884$)$ & $\mathrm{I}-2$ & Rabbit & - & No rise \\
\hline Müller & (1889) & $\begin{array}{l}\text { Ophthalmic } \\
\text { vein occlusion }\end{array}$ & Horse & - & No rise \\
\hline \multirow[t]{2}{*}{ Koster } & (1895) & All & Rabbit & $\begin{array}{l}\text { Immediate } \\
30 \text { min. } \\
2-3 \mathrm{hrs} \\
\text { I day } \\
\text { I }-3 \text { wks } \\
4 \text { mths }\end{array}$ & $\begin{array}{l}\text { Up to } 70 \mathrm{~mm} . \mathrm{Hg} \\
\text { Further rise } \\
\text { approx. } 65 \mathrm{~mm} . \mathrm{Hg} \\
\text { A little lower } \\
\text { Hypotony } \\
\text { Subnormal ( } 14 \mathrm{~mm} . \mathrm{Hg} \text { in one) }\end{array}$ \\
\hline & & $2-3$ & Rabbit & $\begin{array}{l}\text { Immediate } \\
\text { I st day } \\
\text { Later }\end{array}$ & $\begin{array}{l}\text { Normal or raised } \\
\text { Mostly raised } \\
\text { Variable: first hypotony } \\
\rightarrow \text { normal }\end{array}$ \\
\hline \multirow{3}{*}{$\begin{array}{l}\text { Sachsenweger and } \\
\text { Lukoff }\end{array}$} & (1959) & All & Dog & Immediate & $40-80 \mathrm{~mm} . \mathrm{Hg}$ \\
\hline & & $2-3$ & Dog & Immediate & Occasional and transient rise \\
\hline & & $\mathbf{I}$ & Dog & Immediate & No rise \\
\hline Huggert & (1957) & All & Rabbit & $\begin{array}{l}\text { Immediate } \\
\text { Few min. } \\
6 \text { mths }\end{array}$ & $\begin{array}{l}70-100 \mathrm{~mm} . \mathrm{Hg} \\
40-50 \mathrm{~mm} . \mathrm{Hg} \\
\text { In four eyes up to } 30-50 \mathrm{~mm} \text {. } \\
\mathrm{Hg} \\
\text { In other eyes unstable }\end{array}$ \\
\hline Hirata & (r969) & All & Rabbit & $\begin{array}{l}\text { Immediate } \\
\text { Few days }\end{array}$ & $\begin{array}{l}\text { Rise } \\
\text { Normal }\end{array}$ \\
\hline
\end{tabular}


that it returned to near normal in eyes with occlusion of all the VVs and to unrecordably low levels in eyes with occlusion of three VVs; the eyes with occlusion of all the veins dropped to an unrecordable level in 2 weeks. Having reached the lowest level, the IOP started to recover; this occurred within 2 weeks after occlusion of three VVs. In eyes with occlusion of four VVs, however, the recovery of the IOP depended upon the state of the AG, i.e. if the AC was absent the eye had permanent marked hypotony, but if the AC was present the recovery started after an interval of more than 2 weeks. The IOP in these eyes did not recover completely but remained subnormal during the period of follow-up. Histological studies showed evidence of atrophy of the ciliary processes and ciliary body in these eyes (Fig. I0, overleaf).

I R IS

The iris was markedly hyperaemic soon after the occlusion of all or three VVs. With occlusion of one to three VVs, there was sectoral hyperaemia of the iris corresponding to the occluded VVs. The findings of Leber (1873), Koster (1895), and Sachsenweger and Lukoff (I959) were similar.

On follow-up, in the three eyes with all the veins occluded and an absent AC, there were anterior and posterior synechiae with iris atrophy and rubeosis; the clinical appearance resembling that of an eye with long-standing chronic anterior uveitis. In one of the eyes with three VVs occluded, posterior synechiae and sectoral iris atrophy were present. Koster (I895) also found these changes in eyes after occlusion of all the VVs in rabbits.

\section{LENS}

In all the eyes the lens, soon after occlusion of the various VVs, had a proteinous deposit on its anterior surface. During a follow-up period of up to 4 weeks, the deposit cleared without leaving any lens abnormalities in any eye except those with all the VVs occluded. During follow-up, all the eyes with all the VVs occluded showed pigment deposit on the anterior lens surface; in three of these eyes with an absent AC and permanent ocular hypotony, the lens became completely cataractous within 5 weeks (Fig. I $b$ ). Koster ( 1895 ) reported the development of lens opacities in only those eyes which had all the VVs occluded; the changes appeared at first in the anterior cortex of the lens and later resulted in a complete cataract 14, 19, 38, and 9o days after the occlusion. The cataractous change is evidently secondary to impaired nutrition due to degeneration of the ciliary epithelium and ciliary body, as shown by histological studies.

\section{CO R N E A}

Epithelial oedema was seen when the IOP was very high in eyes with all or three VVs occluded. On follow-up, stromal clouding and vascularization by radial vessels from the limbus was seen in three eyes with all the VVs occluded. Two of these had no AC. Koster ( I 895) also noticed a similar corneal vascularization after occlusion of all the VVs and likened it to that of interstitial keratitis, with a gradually progressive pannus from the limbus towards the centre. We feel that the corneal changes are secondary to the changes in the IOP and AC.

The degenerative changes seen in the lens and anterior segment of the eye in these monkeys (particularly in those with occlusion of all the VVs) resemble very much those seen in patients with long-standing anterior uveitis. Almost all the pathological changes in the anterior segment of the monkey eyes depend upon the changes in the AG (e.g. protein-rich aqueous, hyphaema, shallow or absent AC), which in turn are secondary to marked disturbances of the uveal circulation associated with the changes in the ciliary processes and the forward displacement of the iris-lens diaphragm. 


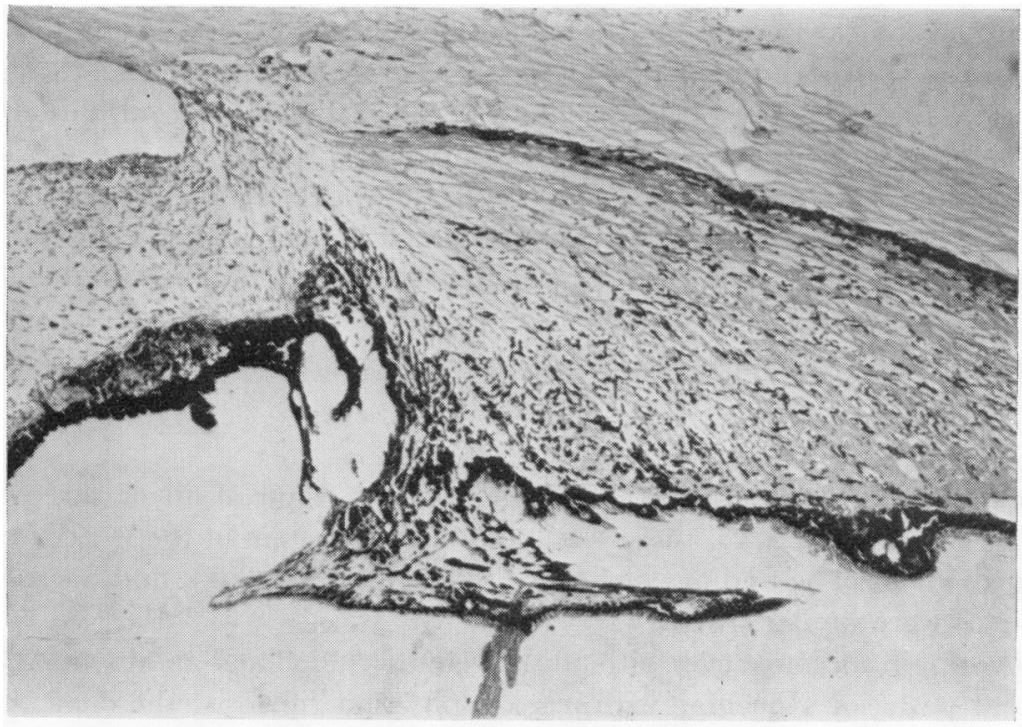

(a)

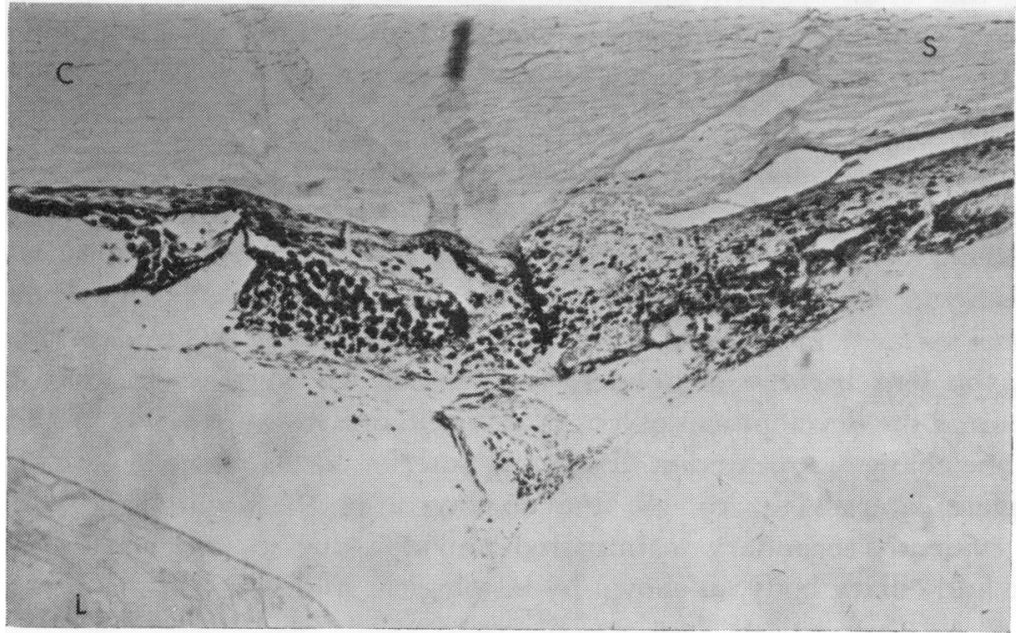

(b)

FIG. Io Photomicrographs of anterior segment of eyes after occlusion of all VVs

(a) 98 days after occlusion, showing degenerative changes in ciliary processes and ciliary body. Haematoxylin and eosin. $\times 123$

(b) $9^{\mathrm{I}}$ days after occlusion, showing very marked degeneration of ciliary body, ciliary processes, and iris, total anterior synechiae of the degenerate iris, and cataractous lens. $\mathrm{C}=$ Cornea; $\mathrm{L}=$ Lens; $\mathrm{S}=$ Sclera. Haematoxylin and eosin. $\times 200$

\section{Clinical significance}

The finding in the anterior chamber after VV occlusion of thick flare or thick gelatinous deposit and hyperaemia of the iris in these eyes mimics perfectly the syndrome diagnosed in patients as non-granulomatous iritis of sudden onset. It is probable that some of the patients in the latter category might, in fact, be cases of occlusion of one VV or more. Intravenous fluorescein injection can be a useful test in such cases to diagnose and determine whether VV occlusion has occurred. Similarly, some of the patients with obscure hyphaema of sudden onset could belong to this category. 
The important role played by interference with the circulation of the vortex veins in some of the major complications following retinal detachment surgery has not been adequately dealt with in the literature so far. Among the complications which are of particular interest in this context are glaucoma, anterior uveitis, necrosis of the anterior segment, and choroidal and intraocular haemorrhages. Most of these complications are usually consequent on the encircling procedure and are aggravated by the application of diathermy or cryopexy. The encircling band would, by invagination and compression, not only reduce the volume of the posterior compartment of the eye (and hence raise the intraocular pressure) but also compress and occlude the choroidal vessels (particularly the thin-walled veins). This must produce marked venous congestion in the anterior part of the uveal tract (i.e. the iris, ciliary body, and anterior part of the choroid). Since (a) the exit of the vortex veins lies posterior to the encircling band, $(b)$ there is no free communication between the anterior and posterior ciliary arteries, and $(c)$ the venous drainage from the part supplied by the anterior ciliary arteries is provided almost entirely by the vortex veins, the amount of venous congestion produced depends upon the degree of strangulation produced by the band and the area involved (e.g. the more posterior the encircling band, the greater will be the area involved). The venous congestion causes marked engorgement of the big thin-walled choroidal veins and also of the loops of blood vessels in the ciliary processes. The ciliary body may also be swollen because of this venous congestion. All these changes increase the volume of the posterior compartment of the eye, and at the same time the capacity of the posterior compartment is decreased by the encircling band: therefore the iris-lens diaphragm is pushed forward, producing a shallow anterior chamber and glaucoma. This will be particularly marked in eyes where the anterior chamber is shallow and has a narrow angle under normal circumstances.

Marked venous engorgement in the choroid in these cases leads to choroidal and even vitreous haemorrhages. Congestion of the vessels in the ciliary processes leads to the production of protein-rich aqueous which, in turn, causes signs resembling those of anterior uveitis, e.g. flare in the anterior chamber, and even the deposition of gelatinous material in the anterior chamber in severe cases (which may be confused with endophthalmitis). We feel that the so-called uveitis seen after retinal detachment surgery is, in fact, a part of this syndrome and not a true uveitis. Intravenous injection of fluorescein soon after detachment surgery should produce in such a case immediate and marked leakage of the dye from the posterior into the anterior chamber (Fig. I I, overleaf), indicating uveal venous congestion.

Microscopic hyphaema can occur and in severe cases frank hyphaema may be presentdepending upon the degree of venous congestion in the ciliary processes. In very marked venous stasis of the anterior uveal tract, anterior segment necrosis would supervene.

When a sectoral scleral indentation is made to produce a buckle in one sector and is accompanied by the application of diathermy or cryopexy, this would interfere with the outflow of one or more vortex veins and thus produce changes similar to those seen after occlusion of one or more of the vortex veins (p. 226) -in mild cases the signs of anterior uveitis.

Thus, in retinal detachment surgery, interference with the venous drainage from the anterior half or more of the uveal tract can be the source of many serious complications. As a safety precaution, it is suggested that in all these cases the following investigations should be carried out immediately after the operation:

(i) Slit-lamp examination of the anterior chamber: for protein-rich aqueous and depth of the anterior chamber. 

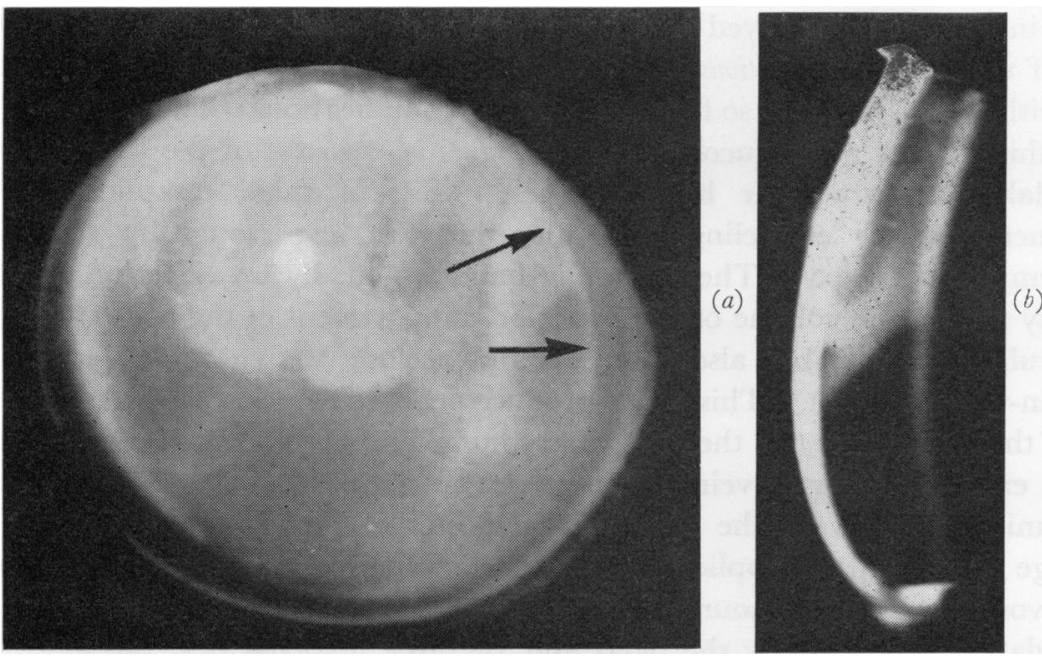

FIG. I I Fluorescent photographs of anterior segment of an eye $2 \frac{1}{4}$ hrs after occlusion of superior temporal and nasal VVs in a rhesus monkey (about Io to I 5 minutes after intravenous injection of fluorescein)

(a) Ordinary view Note fluorescence in upper part of anterior chamber, as well as in (a) seepage of

(b) Slit-lamp view $\int$ fluorescein from posterior chamber into anterior chamber (arrow)

(ii) Intravenous fluorescein test: the dye is injected intravenously and its leakage into the anterior chamber from the posterior chamber is studied for 15 minutes after the injection. A large amount of fluorescein seeping into the anterior chamber from the posterior chamber via the pupil (Fig. I I) indicates embarrassment of the anterior uveal venous drainage.

(iii) Estimation of the intraocular pressure: a very high intraocular pressure, even if it is below the level required to produce pulsation of the central retinal artery on the optic disc, can interfere with the blood supply of the optic disc (Hayreh, I969, I970; Hayreh and others, 1970) and, in older people, may even produce ischaemic optic neuropathy (Hayreh and Baines, 1972b) with permanent total or partial visual loss.

Some workers have speculated that interference with the choroidal venous drainage from the macular area may be responsible for the production of subretinal haemorrhage in disciform macular degeneration. As mentioned above (p. 227), the meeting point of the distribution of the four vortex veins is situated slightly nasal to the foveal region, so that the choroidal veins tend to radiate away from the foveal area (or, to describe the picture in another way, they meet one another close to the fovea). It is very tempting to postulate under these circumstances that a sudden occlusion of one of the vortex veins in patients with unhealthy vessels could initiate choroidal haemorrhage in the macular region. However, in the present study, occlusion of one or more vortex veins did not produce any macular pathology. In one eye (not included in this series) occlusion of the superior temporal vortex vein combined with occlusion of one of the many short posterior ciliary arteries in that region produced a subretinal macular haemorrhage, originating from the chorio-capillaris and clinically resembling the subretinal haemorrhage seen in macular degeneration. However, on repeating the experiment in six more eyes, no such haemorrhage was seen! It is therefore not productive to base speculations as to the effects of vortex vein occlusion in old and arteriosclerotic human eyes on results obtained from the eyes of young and healthy monkeys. With the evidence so far available, we find no relationship between senile macular degeneration and vortex vein occlusion. 


\section{Conclusions}

(I) In rhesus monkeys, the VVs do not communicate freely with one another but have a well-defined distribution in the entire length of the uveal tract, each vein usually draining a quadrant of the uvea. Because of this segmental distribution, and the segmental distribution of the posterior ciliary arteries and of the chorio-capillaris, occlusion of a VV produces marked venous congestion and sluggish circulation in the affected segment of the uveal tract. The circulation is restored to normal in I to 2 weeks or longer depending upon the number of VVs occluded.

(2) No significant fundus lesions are seen. Chorio-retinal degenerative lesions were seen on follow-up near the equator in the region of the occluded $\mathrm{VV}$, even if only one vein was occluded.

(3) The ciliary processes bear the main brunt of the VV occlusion because of marked engorgement of the subepithelial capillaries in the occluded sector. On occlusion of the VVs, instead of the normal aqueous production, marked exuda:ion of protein-rich aqueous and profuse leakage of fluorescein is seen from the sector drained by the occluded VV. This produces a gelatinous deposit on the anterior surface of the lens and in the AC. On occlusion of two or three or all the VVs, in addition to the protein-rich aqueous, haemorrhages due to rupture of the subepithelial capillaries produce hyphaema-the more VVs are occluded, the more common this consequence is. On follow-up, atrophy of the ciliary processes develops, the extent of involvement depending upon the number of VVs occluded.

(4) On occlusion of three or all VVs, forward movement of the iris-lens diaphragm leads to a shallow AC. On follow-up, the AC was found to be permanently absent in half of the eyes with occlusion of all the VVs.

(5) On occlusion of three or all the VVs, there is a sudden rise in the IOP. However, I to 2 weeks after occlusion there is marked ocular hypotony. The IOP improves slowly after that, but was never found to have recovered to normal during the period of follow-up in this study.

(6) Immediately after the occlusion, the iris shows sectoral hyperaemia corresponding to the occluded VV, which is most marked after the occlusion of all or three VVs.

(7) Occlusion of all the VVs later resulted in iris atrophy with anterior and posterior synechiae, cataractous lens, and vascularized cornea in half of the eyes on follow-up. Almost all these eyes had absent AG and permanent ocular hypotony.

(8) Clinically, VV occlusion can mimic non-granulomatous anterior uveitis. In some of the cases with VV occlusion, particularly those with occlusion of three or all VVs, the syndrome of anterior segment ischaemia could be thought to be present because of the production of marked disturbances in the uveal circulation in them.

(9) Interference with the circulation in the VVs after retinal detachment surgery seems to play an important role in producing some of the major complications of surgery, e.g. glaucoma, anterior uveitis, necrosis of the anterior segment, and choroidal and intraocular haemorrhages.

\section{Summary}

In forty eyes of rhesus monkeys, the various vortex veins (VVs) were cauterized outside the globe close to their exit in various combinations, i.e. one, two, three, or all the VVs. 
Eighteen eyes were followed for periods of up to 3 months. The occlusion produced immediate venous congestion and stasis in the segment of the uveal tract drained by the occluded vein. The vessels in the ciliary processes were most affected by the occlusion which resulted in the production of protein-rich aqueous and, on occlusion of two, three, or all VVs, additional haemorrhages were seen, resulting in hyphaemata-the more VVs were occluded, the morefrequent were the hyphaemata. In cases with occlusion of three or all VVs forward movement of the iris-lens diaphragm was associated with raised intraocular pressure and a shallow or absent anterior chamber; these eyes later developed degenerative changes, e.g. iris atrophy with anterior and posterior synechiae, cataractous lens, vascularized cornea, and atrophy of the ciliary processes with ocular hypotony. On follow-up, normal uveal circulation was re-established after I or 2 weeks or longer, depending upon the number of VVs occluded. Clinically, VV occlusion can mimic non-granulomatous anterior uveitis and some of these cases resemble the syndrome of anterior segment ischaemia. The pathogenesis of some of the major complications of retinal detachment surgery is discussed in the light of the findings.

We are grateful to Dr. Anne Schloss for her invaluable help in translating the German literature, without which the review of the literature would not have been possible. We should also like to express our gratitude to Mrs. S. B. Hayreh for her help in the preparation of the manuscript, to Mr. Alasdair McDonald for the illustrations, and to Mrs. Anne Roger for her secretarial assistance.

\section{References}

ADAmiuk, E. (1867) Ann. Oculist. (Paris), 58, I ARLT, F. (1884) 'Zur Lehre vom Glaukom', p. I I 3. Braumüller, Vienna COGAN, J. F. (1960) Brit. 7. Ophthal., 44, 185 DE FARIA and silva, D. (1950) Ann. Oculist. (Paris), 183, 400 HAYREH, s. s. (1964) Exp. Eye Res., 3, I6

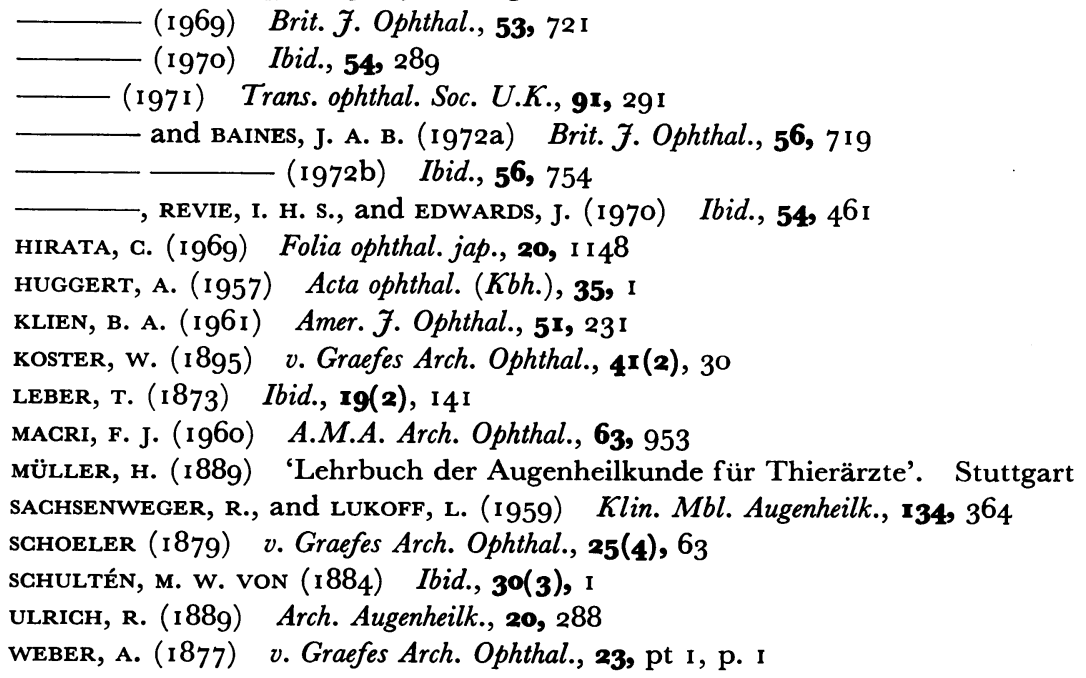

